\title{
Fixed Point Theorems on Some New Types of Cyclic Contractions and Their Generalization
}

\author{
Dev Raj Joshi ${ }^{1}$, Piyush Kumar Tripathi ${ }^{2}$, Chet Raj Bhatta ${ }^{3}$ \\ 1 Amity School of Applied Science, Amity University Lucknow Campus,U.P. \\ 2 Department of Mathematics, Amity School of Applied Science, Amity University Lucknow Campus, U.P. \\ 3 Central Department of Mathematics,T.U. \\ Correspondence to: Dev Raj Joshi, Email: devrajsir@gmail.com
}

\begin{abstract}
There are different types of contraction in the existing literature for the generalization of Banach's contraction principle. Our aim in this paper is to generalize cyclic contraction so that it can explain all types of cyclic contraction as a particular case. Besides all contractions in the existing literature we introduce some new types of cyclic contraction before defining the generalized cyclic contraction.
\end{abstract}

Keywords: Cyclic contraction, Kannan type cyclic contraction, Chatterjee type cyclic contraction, Reich type cyclic contraction, Ciric type contraction, Meir and Keeler type cyclic contraction

DOI: https://doi.org/10.3126/jnms.v4i2.41461

\section{Introduction}

After Banach's contraction principle of a self mapping for existence of fixed point published in 1922[11, there are so many generalizations on his work. In 2003 Kirk et al. 12 introduced the concept of cyclic contraction and proved fixed point theorem for cyclic contraction mapping. Erdal et al. [1] in 2011 published best proximity point based on different type of contractions such as cyclic contraction, Kannan type cyclic contraction, Chattejee type cyclic contraction and Reich type cyclic contraction[13, 14, 15]. Recently in 2019, Dev Raj et al.3 find out the Generalized Cyclic Contraction Mapping and its fixed point theorem in Complete Metric Spaces. Other Many authors have investigated many theorems on this field such as Rus and Pacurar[10] introduced some fixed point results for cyclic $\phi$-contractions. Piatek 9 , obtained some results on cyclic Meir-Keeler contractions in metric spaces. Karapinar [4] used fixed point result for weakly contractive map to establish some fixed point results for cyclic weak $\phi$-contraction mappings.In this paper we introduced some new types of cyclic contraction mappings and proved unique fixed point theorem on such cyclic contractions.in the remarks section it is shown that the theorem in 3 is the Generalized theorem of all type of Cyclic contractions.

\section{Preliminaries}

Here are some definitions and theorems.

Definition 2.1. Let $X$ be a non-empty set and a mapping $d: X \times X \rightarrow R^{+}$is called metric in $X$, if it satisfies following conditions

$\left(M_{1}\right) d(x, y) \geq 0$

$\left(M_{2}\right) d(x, y)=0$, if and only if $x=y$

$\left(M_{3}\right) d(x, y)=d(y, x)$, for all $x, y \in X$

$\left(M_{4}\right) d(x, y) \leq d(x, z)+d(z, y)$, for all $x, y, z \in X$. Then the set $X$

with metric $d$ is called metric space and it is denoted by $(X, d)$

Definition 2.2. Let $A$ and $B$ be nonempty subsets of a metric space $(X, d)$. A mapping $f: A \cup B \rightarrow A \cup B$ is called cyclic if $f(A) \subset B$ and $f(B) \subset A$.

Definition 2.3. [12] Let $A$ and $B$ be non-empty subsets of a metric space $(X, d)$. A mapping $f: A \cup B \rightarrow$ $A \cup B$ is called cyclic contraction if $f$ is cyclic and there exist $P \in(0,1)$ such that $d(f x, f y) \leq P d(x, y)$ for all $x \in A$ and $y \in B$. 
Theorem 2.1. [12] Let $A$ and $B$ be non-empty closed subsets of a complete metric space $(X, d)$. If a mapping $f: A \cup B \rightarrow A \cup B$ be cyclic contraction in $X$ then $f$ has a unique fixed point in $A \cap B$

Definition 2.4. [1] Let $A$ and $B$ be non-empty subsets of a metric space $(X, d)$. A mapping $f: A \cup$ $B \rightarrow A \cup B$ is called Kannan type cyclic contraction if $f$ is cyclic and there exist $q \in(0,1 / 2)$ such that $d(f x, f y) \leq q[d(f x, x)+d(f y, y)]$ for all $x \in A$ and $y \in B$.

Theorem 2.2. [1] Let $A$ and $B$ be non-empty closed subsets of a complete metric space $(X, d)$.If a mapping $f: A \cup B \rightarrow A \cup B$ be Kannan type cyclic contraction in $X$ then $f$ has a unique fixed point in $A \cap B$.

Definition 2.5. [1] Let $A$ and $B$ be non-empty subsets of a metric space $(X, d)$. A mapping $f: A \cup$ $B \rightarrow A \cup B$ is called Tetterjee type cyclic contraction if $f$ is cyclic and there exist $r \in(0,1 / 2)$ such that $d(f x, f y) \leq q[d(f x, y)+d(f y, x)]$ for all $x \in A$ and $y \in B$.

Theorem 2.3. [1] Let $A$ and $B$ be non-empty closed subsets of a complete metric space $(X, d)$.If a mapping $f: A \cup B \rightarrow A \cup B$ be Chetterjee type cyclic contraction in $X$ then $f$ has a unique fixed point in $A \cap B$.

Definition 2.6. [1] Let $A$ and $B$ be non-empty subsets of a metric space $(X, d)$. A mapping $f: A \cup$ $B \rightarrow A \cup B$ is called Reich type cyclic contraction if $f$ is cyclic and there exist $k \in(0,1 / 3)$ such that $d(f x, f y) \leq k[d(x, y)+d(f x, x)+d(f y, y)]$ for all $x \in A$ and $y \in B$.

Theorem 2.4. [1] Let $A$ and $B$ be non-empty closed subsets of a complete metric space $(X, d)$.If a mapping $f: A \cup B \rightarrow A \cup B$ be Reich type cyclic contraction in $X$ then $f$ has a unique fixed point in $A \cap B$.

Definition 2.7. [3] Let $A$ and $B$ be non-empty subsets of a metric space $(X, d)$. A mapping $f: A \cup$ $B \rightarrow A \cup B$ is called Generalized cyclic contraction if $f$ is cyclic and there exist $p, q, r \in(0,1)$ satisfying $p+2 q+2 r<1$ such that

$d(f x, f y) \leq p d(x, y)+q[d(f x, x)+d(f y, y)]+r[d(f x, y)+d(f y, x)]$ For all $x \in A$ and $y \in B$.

Theorem 2.5. [3] Let $A$ and $B$ be non-empty closed subsets of a complete metric space $(X, d)$.If a mapping $f: A \cup B \rightarrow A \cup B$ be generalized cyclic contraction in $X$ then $f$ has a unique fixed point in $A \cap B$. The proof is in [3].

\section{Main Results}

Here we introduced three new types of cyclic contractions and name them type $C_{1}, C_{2}$ and $C_{3}$.

Definition 3.1. Let $A$ and $B$ are non-empty subsets of a metric space $(X, d)$. A mapping $f: A \cup B \rightarrow A \cup B$ is called $C_{1}$ type cyclic contraction if $f$ is cyclic and there exist $c \in(0,1 / 3)$ such that $d(f x, f y) \leq c[d(x, y)+$ $d(f x, y)+d(f y, x)]$ for all $x \in A$ and $y \in B$.

Theorem 3.1. Let $A$ and $B$ are non-empty closed subsets of a complete metric space $(X, d)$.If a mapping $f: A \cup B \rightarrow A \cup B$ be $C_{1}$ type cyclic contraction inX then $f$ has a unique fixed point in $A \cap B$.

Proof. Fix $x \in A$. Now by the definition of 3.1 there exist $c \in(0,1 / 3)$ such that,

$$
\begin{aligned}
d\left(f^{2} x, f x\right) & =d(f(f x), f x) \\
& \leq c\left[d(f x, x)+d\left(f^{2} x, x\right)+d(f x, f x)\right] \\
& \leq c\left[d(f x, x)+d\left(f^{2} x, f x\right)+d(f x, x)\right] \\
& \leq 2 c d(f x, x)+c d\left(f^{2} x, f x\right)
\end{aligned}
$$

Therefore,

$$
\begin{aligned}
(1-c) d\left(f^{2} x, f x\right) & \leq 2 c d(f x, x) \\
\text { or, } d\left(f^{2} x, f x\right) & \leq \frac{2 c}{1-c} d(f x, x) \\
\text { or, } d\left(f^{2} x, f x\right) & \leq \sigma d(f x, x)
\end{aligned}
$$


Since $c \in(0,1 / 3)$ so $\sigma=\frac{2 c}{1-c}<1$

Now by induction we have $d\left(f^{n+1} x, f^{n} x\right) \leq \sigma^{n} d(f x, x)$ Then, for any $m, n \in N$ where $n>m$ we have

$$
\begin{aligned}
d\left(f^{n} x, f^{m} x\right) & \leq d\left(f^{n} x, f^{n-1} x\right)+d\left(f^{n-1} x, f^{n-2} x\right)+\ldots+d\left(f^{m+1} x, f^{m} x\right) \\
& \leq\left(\sigma^{n-1}+\sigma^{n-2}+\ldots+\sigma^{m+1}+\sigma^{m}\right) d(f x, x) \\
& \leq \sigma^{m}\left(\sigma^{n-m-1}+\sigma^{n-m-2}+\ldots+\sigma+1\right) d(f x, x) \\
& \leq \sigma^{m} \frac{1}{1-\sigma} d(f x, x) \quad[\text { Sum of infinite series as, } n \rightarrow \infty]
\end{aligned}
$$

Since $\sigma<1$ so $\sigma^{m} \rightarrow 0$ as, $m \rightarrow \infty$ thus, we have $d\left(f^{n} x, f^{m} x\right) \rightarrow 0$ as $n, m \rightarrow \infty$. Therefore $\left\langle f^{n} x\right\rangle$ is Cauchy sequence. Since $(X, d)$ is complete so $\left\langle f^{n} x\right\rangle$ converges to some point $u \in X$. Since $x \in A$ so $f^{2 n} x \in A$ and $f^{2 n-1} x \in B$. But $f^{2 n} x \rightarrow u$ and $f^{2 n-1} x \rightarrow u$. As the sets are closed so $u \in A \cap B$.It remains to show that $f u=u$ we have,

$$
\begin{aligned}
d\left(f u, f^{2 n} x\right) & =d\left(f u, f\left(f^{2 n-1} x\right)\right) \\
& \leq c\left[d\left(u, f^{2 n-1} x\right)+d\left(f u, f^{2 n-1} x\right)+d\left(f^{2 n} x, u\right)\right]
\end{aligned}
$$

Taking, $n \rightarrow \infty$, we get $f^{2 n} x=u$ and $f^{2 n-1} x=u$, then we have,

$$
\begin{array}{ll} 
& d(f u, u) \leq c[d(u, u)+d(f u, u)+d(u, u)] \\
\Longrightarrow \quad & d(f u, u) \leq c d(f u, u) \quad[\because d(u, u)=0] \\
\Longrightarrow \quad & (1-c) d(f u, u) \leq 0
\end{array}
$$

Since $1-c>0$ so $d(f u, u)=0$

$\Longrightarrow f u=u$

Thus $u$ is a fixed point.To show the uniqueness of fixed point, let us suppose that there exist two fixed points $u$ and $v$ such that $f u=u$ and $f v=v$ Then we have,

$$
\begin{aligned}
d(f u, f v) & \leq c[d(u, v)+d(f u, v)+d(u, f v)] \\
& \leq c[d(u, v)+d(u, v)+d(u, v) \\
& \leq 3 c d(u, v) \\
\text { or, } d(u, v) & \leq 3 c d(u, v) \\
\text { or, }(1-3 c) d(u, v) & \leq 0 \\
\Longrightarrow d(u, v) & =0 \quad[\because(1-3 c)>0] \\
\Longrightarrow u & =v .
\end{aligned}
$$

Therefore $f$ has a unique fixed point in $A \cap B$, this completes the proof.

Definition 3.2. Let $A$ and $B$ be non-empty subsets of a metric space $(X, d)$. A mapping $f: A \cup B \rightarrow A \cup B$ is called $C_{2}$ type cyclic contraction if $f$ is cyclic and there exist $c \in\left(0, \frac{1}{4}\right)$ such that $d(f x, f y) \leq c[d(f x, x)+$ $d(f y, y)+d(f x, y)+d(f y, x)]$ for all $x \in A$ and $y \in B$.

Theorem 3.2. Let $A$ and $B$ be non-empty closed subsets of a complete metric space $(X, d)$.If a mapping $f: A \cup B \rightarrow A \cup B$ be $C_{2}$ type cyclic contraction in $X$ then $f$ has a unique fixed point in $A \cap B$. 
Proof. Fix $x \in A$. Now by the definition of 3.2 there exist $c \in\left(0, \frac{1}{4}\right)$ such that

$$
\begin{aligned}
d\left(f^{2} x, f x\right) & =d(f(f x), f x) \\
& \leq c\left[d\left(f^{2} x, f x\right)+d(f x, x)+d\left(f^{2} x, x\right)+d(f x, f x)\right] \\
& \leq c\left[d\left(f^{2} x, f x\right)+d(f x, x)+d\left(f^{2} x, f x\right)+d(f x, x)\right] \\
{[\because d(f x, f x)=0] } & \leq 2 c d(f x, x)+2 c d\left(f^{2} x, f x\right) \\
\text { Or }(1-2 c) d\left(f^{2} x, f x\right) & \leq 2 c d(f x, x) \\
\text { Ord } d\left(f^{2} x, f x\right) & \leq \frac{2 c}{1-2 c} d(f x, x) \\
\text { Ord } d\left(f^{2} x, f x\right) & \leq \alpha d(f x, x)
\end{aligned}
$$

Now by induction we have $d\left(f^{n+1} x, f^{n} x\right) \leq \alpha^{n} d(f x, x)$ Then, for any $m, n \in N$ where $n>m$ we have

$$
\begin{aligned}
d\left(f^{n} x, f^{m} x\right) & \leq d\left(f^{n} x, f^{n-1} x\right)+d\left(f^{n-1} x, f^{n-2} x\right)+\ldots+d\left(f^{m+1} x, f^{m} x\right) \\
& \leq\left(\alpha^{n-1}+\alpha^{n-2}+\ldots+\alpha^{m+1}+\alpha^{m}\right) d(f x, x) \\
& \leq \alpha^{m}\left(\alpha^{n-m-1}+\alpha^{n-m-2}+\ldots+\alpha+1\right) d(f x, x) \\
& \left.\leq \alpha^{m} \frac{1}{1-\alpha} d(f x, x) \quad \text { [Sumofinfiniteseriesas, } n \rightarrow \infty\right]
\end{aligned}
$$

Since $\alpha<1$ so $\alpha^{m} \rightarrow 0$ as $m \rightarrow \infty$ thus, we have $d\left(f^{n} x, f^{m} x\right) \rightarrow 0$ as $n, m \rightarrow \infty$ Therefore $\left\langle f^{n} x\right\rangle$ is Cauchy sequence. Since $(X, d)$ is complete so $\left\langle f^{n} x\right\rangle$ converges to some point $u \in X$. Since $x \in A$ so $f^{2} n x \in A$ and $f^{2 n-1} x \in B$. But $f^{2 n} \rightarrow u$ and $f^{2 n-} x \rightarrow u$. As the sets are closed so $u \in A \cap B$.It remains to show that $f u=u$ we have,

$$
\begin{aligned}
d\left(f u, f^{2 n} x\right) & \left.\leq d\left(f u, f\left(f^{2 n}-1\right) x\right)\right) \\
& \leq c\left[d(f u, u)+d\left(f^{2 n} x, f^{2 n-1} x\right)+d\left(f u, f^{2 n-1} x\right)+d\left(f^{2 n} x, u\right)\right]
\end{aligned}
$$

Taking, $n \rightarrow \infty$, we get $f^{2 n} x=u$ and $f^{2 n-1} x=u$, then we have

$$
\begin{aligned}
d(f u, u) & \leq c[d(f u, u)+d(u, u)+d(f u, u)+d(u, u)] \\
\text { Or } d(f u, u) & \leq 2 c d(f u, u) \\
\text { Or }(1-2 c) d(f u, u) & \leq 0 \\
\text { Since } 1-2 c>0 \text { so } d(f u, u) & =0 \\
\Longrightarrow f u & =u
\end{aligned}
$$

Thus $u$ is a fixed point.To show the uniqueness of fixed point, let us suppose that there exist two fixed points $u$ and $v$ such that $f u=u$ and $f v=v$ Then we have,

$$
\begin{aligned}
d(f u, f v) & \leq c[d(f u, u)+d(f v, v)+d(f u, v)+d(f v, u)] \\
& \leq c[d(u, u)+d(v, v)+d(u, v)+d(v, u)] \\
& \leq 2 c d(u, v) \\
\text { Or } d(u, v) & \leq 2 c d(u, v) \\
\text { Or }(1-2 c) d(u, v) & \leq 0 \\
\Longrightarrow(u, v) & =0 \quad[\because 1-2 c>0] \\
\Longrightarrow u & =v .
\end{aligned}
$$

Therefore $f$ has a unique fixed point in $A \cap B$, this complete the proof.

Definition 3.3. Let $A$ and $B$ are non-empty subsets of a metric space $(X, d)$. A mapping $f: A \cup B \rightarrow A \cup B$ is called $C_{3}$ type cyclic contraction if $f$ is cyclic and there exist $c \in\left(0, \frac{1}{5}\right)$ such that $d(f x, f y) \leq c[d(x, y)+$ $d(f x, x)+d(f y, y)+d(f x, y)+d(f y, x)]$ for all $x \in A$ and $y \in B$. 
Theorem 3.3. Let $A$ and $B$ be non-empty closed subsets of a complete metric space $(X, d)$.If a mapping $f: A \cup B \rightarrow A \cup B$ be $C_{3}$ type cyclic contraction inX then $f$ has a unique fixed point in $A \cap B$.

Proof. Fix $x \in A$. Now by the definition of 3.3 there exist $c \in\left(0, \frac{1}{5}\right)$ such that,

$$
\begin{aligned}
d\left(f^{2} x, f x\right) & =d(f(f x), f x) \\
& \leq c\left[d(f x, x)+d\left(f^{2} x, f x\right)+d(f x, x)+d\left(f^{2} x, x\right)+d(f x, f x)\right] \\
& \leq c\left[d(f x, x)+d\left(f^{2} x, f x\right)+d(f x, x)+d\left(f^{2} x, f x\right)+d(f x, x)\right] \\
{[\because(f x, f x)=0] } & \leq 3 c d(f x, x)+2 c d\left(f^{2} x, f x\right) \\
\Longrightarrow(1-2 c) d\left(f^{2} x, f x\right) & \leq 3 c d(T x, x) \\
\Longrightarrow d\left(f^{2} x, f x\right) & \leq \frac{3 c}{1-2 c} d(f x, x) \\
\Longrightarrow d\left(f^{2} x, f x\right) & \leq \gamma d(f x, x)
\end{aligned}
$$

Since $c \in\left(0, \frac{1}{5}\right)$ so $\gamma=\frac{3 c}{1-2 c}<1$ Now by induction we have $d\left(f^{n+1} x, f^{n} x\right) \leq \gamma^{n} d(f x, x)$ Then for any $m, n \in N$ where $n>m$ we have,

$$
\begin{aligned}
d\left(f^{n} x, f^{m} x\right) & \leq d\left(f^{n} x, f^{n-1} x\right)+d\left(f^{n-1} x, f^{n-2} x\right)+\ldots+d\left(f^{m+1} x, f^{m} x\right) \\
& \leq\left(\gamma^{n-1}+\gamma^{n-2}+\ldots+\gamma^{m+1}+\gamma^{m}\right) d(f x, x) \\
& \leq \gamma^{m}\left(\gamma^{n-m-1}+\gamma^{n-m-2}+\ldots+\gamma+1\right) d(f x, x) \\
& \leq \gamma^{m} \frac{1}{1-\gamma} d(f x, x) \quad[\because \text { Sum of infinite series as }, m \rightarrow \infty]
\end{aligned}
$$

Since $\gamma<1$ so $\gamma^{m} \rightarrow 0$ as $n, m \rightarrow \infty$ thus, we have $d\left(f^{n} x, f^{m} x\right) \rightarrow 0$ as $n, m \rightarrow$

infty Therefore $\left\langle f^{n} x\right\rangle$ is Cauchy sequence. Since $(X, d)$ is complete so $\left\langle f^{n} x\right\rangle$ converges to some point $u \in X$. Since $x \in A$ so $f^{2 n} x \in A$ and $f^{2 n-1} x \in B$. But $f^{2 n} \rightarrow u$ and $f^{2 n-1} x \rightarrow u$. As the sets are closed so $u \in A \cap B$.It remains to show that $f u=u$ we have,

$$
\begin{aligned}
d\left(f u, f^{2 n} x\right) & =d\left(f u, f\left(f^{2 n-1} x\right)\right) \\
& \leq c\left[d\left(u, f^{2 n-1} x\right)+d(f u, u)+d\left(f^{2 n} x, f^{2 n-1} x\right)+d\left(f u, f^{2 n-1} x\right)+d\left(f^{2 n} x, u\right)\right]
\end{aligned}
$$

Taking, $n \rightarrow \infty$, we get $f^{2 n} x=u$ and $f^{2 n-1} x=u$, then we have,

$$
\begin{aligned}
d(f u, u) & \leq c[d(u, u)+d(f u, u)+d(u, u)]+d(f u, u)+d(u, u)] \\
\text { or, } d(f u, u) & \leq 2 c d(f u, u) \\
\text { or, }(1-2 c) d(f u, u) & \leq 0 \\
\text { Since, } 1-2 c>0, d(f u, u) & =0 \Longrightarrow f u=u
\end{aligned}
$$

Thus $u$ is a fixed point.To show the uniqueness of fixed point, let us suppose that there exist two fixed points $u$ and $v$ such that $f u=u$ and $f v=v$ Then we have,

$$
\begin{aligned}
d(f u, f v) & \leq c[d(u, v)+d(f u, u)+d(f v, v)+d(f u, v)+d(f v, u)] \\
& \leq c[d(u, v)+d(u, u)+d(v, v)+d(u, v)+d(v, u)] \\
& \leq 3 c d(u, v) \\
\text { or, } d(u, v) & \leq 3 c d(u, v) \\
\text { or, }(1-3 c) d(u, v) & \leq 0 \\
\Longrightarrow(u, v) & =0 \quad[\because 1-3 c>0] \\
\Longrightarrow u & =v .
\end{aligned}
$$

Therefore $f$ has a unique fixed point in $A \cap B$, this complete the proof. 


\section{Remarks}

The cyclic contractions $C_{1}, C_{2}$ and $C_{3}$ are the special cases of Generalized cyclic contraction in [3] and their fixed point theorems also special case of Generalized fixed point theorem in $[3]$ as given below.

- When $q=0$ and $p=r$ in generalized theorem in [3] then it becomes $C_{1}$ type cyclic contraction 3.3.

- When $p=0$ and $q=r$ in generalized theorem in 3 , then it becomes $C_{2}$ type cyclic contraction theorem 3.2 .

- When $p=q=r$ in generalized theorem in [3] then it becomes $C_{3}$ type cyclic contraction theorem 3.1 .

\section{Conclusions}

It is found that the three new types of cyclic contractions $C_{1}, C_{2}$ and $C_{3}$ can be formulated to exist fixed points accordingly and they are also special cases of theorem in 3 as shown in remarks above.

\section{References}

[1] Karapinar, E. and Erhan, I.M., 2011. Best proximity point on different type contractions, Appl. Math. Inf. Sci., 5(3), $558-569$.

[2] Jleli, M., Karapınar, E. and Samet, B., 2012, Fixed point results for almost generalized cyclic-weak contractive type mappings with applications, Abstract and Applied Analysis, 2012, 1 - 17.

[3] Joshi D. R., Tripathi, P.K, Agrawal, A. and Mishra, A., 2019, Generalized cyclic contraction mapping and its unique fixed point theorem in complete metric spaces, International Journal of Advance Science and Technology, 28(20), 231 - 233.

[4] Karapinar, E., 2011, Fixed point theory for cyclic weak $\phi$-contraction, Appl. Math. Lett, 24, $822-$ 825.

[5] Kosuru, G. and Veeramani, P., 2010, Cyclic contractions and best proximity pair theorems, arXiv preprint arXiv:1012.1434.

[6] Karpagam, S., Agrawal, S., 2011, Best proximity point theorems for cyclic orbital Meir-Keeler contraction maps, Nonlinear Anal., 74, 1040 - 1046.

[7] Neammanee, K., Kaewkhao, A., 2011, Fixed points and best proximity points for multi-valued mapping satisfying cyclical condition. Int. J. Math. Sci. Appl, 1(1), 1 - 9.

[8] Petric, M.A., 2011, Best proximity point theorems for weak cyclic Kannan contractions. Filomat, 25(1), $145-154$

[9] Piatek, B., 2011, On cyclic Meir-Keeler contractions in metric spaces, Nonlinear Anal, 74, 35 - 40.

[10] Pacurar, M., Rus, I. A., 2010, Fixed point theory for cyclic $\phi$ - contractions, Nonlinear Anal, 72(3-4), $1181-1187$.

[11] Banach, S., 1922. On operations in abstract sets and their application to integral equations, Fund. Math , 3(1), 133 - 181.

[12] Kirk, W.A., Srinivasan, P.S. and Veeramani, P., 2003. Fixed points for mappings satisfying cyclical contractive conditions, Fixed Point Theory, 4(1), 79 - 89.

[13] Kannan R. (1968) Some Results on Fixed Points, Bulletin Calcutta Mathematical Society, 10, 71 - 76.

[14] Chatterjee S. K., 1972, Fixed point theorem, C. R. Acad, Bulgar Sci. 25, 727 - 730.

[15] Reich, S., 1971, Kannan's fixed point theorem, Bull. Univ. Mat. Italiana, 4(4), 1 - 11. 